\section{Indicadores sócio-demográficos e a epidemia de dengue em 2002 no Estado do Rio de Janeiro, Brasil}

\author{
Socio-demographic factors and the dengue fever \\ epidemic in 2002 in the State of Rio de Janeiro, \\ Brazil
}

\author{
1 Escola Nacional de Saúde \\ Pública Sergio Arouca, \\ Fundação Oswaldo Cruz, \\ Rio de Janeiro, Brasil. \\ 2 Instituto de Estudos em \\ Saúde Coletiva, Universidade \\ Federal do Rio de Janeiro, \\ Rio de Janeiro, Brasil. \\ Correspondência \\ T. R. A. Teixeira \\ Instituto de Estudos em \\ Saúde Coletiva, Universidade \\ Federal do Rio de Janeiro \\ Av. Brigadeiro Trompowsky \\ $s / n$ sala 03, Rio de Janeiro, $R J$ \\ 21949-900, Brasil \\ tatirodriguesaraujo@yahoo. \\ com.br
}

\begin{abstract}
This study analyzed the dengue fever epidemic in 2002 and the socio-demographic context of the State of Rio de Janeiro, Brazil, using spatial analysis and statistical modeling. The incidence rate was calculated for resident dengue cases in the State in 2002. The study analyzed associations between incidence and socio-demographic variables and spatial autocorrelation using the Moran Global Index, which showed spatial dependence for both the outcome and the independent variables. A multivariate linear regression model was used. The variables' proportion of urban population, percentage of the population with running water, and percentage of coverage by the Family Health Program (FHP) explained $30.2 \%$ of the total variance in the epidemic's incidence rate. The model's residuals did not show spatial autocorrelation. The associations were in the expected direction, and the findings are corroborated by other studies that showed higher dengue incidence in areas characterized by growing urbanization and deficient running water and water supply, while highlighting the FHP as an important facilitator of vector control strategies.
\end{abstract}

Dengue; Disease Outbreaks; Spatial Analysis
Tatiana Rodrigues de Araujo Teixeira 1,2 Roberto de Andrade Medronho 2

\section{Introdução}

No Brasil, as condições sócio-ambientais, aliadas à baixa efetividade dos programas de combate ao vetor, favoreceram a grande difusão do Aedes aegypti, desde a sua reintrodução no território nacional a partir do Estado da Bahia, em 1976 1,2,3. A presença do vetor voltou a ser registrada no Estado do Rio de Janeiro ainda em 1976, após trinta e um anos sem ocorrência verificável 4, e há mais de vinte anos o estado vem mantendo-se receptivo e vulnerável à disseminação do vírus do dengue.

Esse vírus possui quatro sorotipos, sendo que três deles tiveram sua introdução e circulação confirmadas no Estado do Rio de Janeiro. A primeira descrição de doença clinicamente semelhante ao dengue no Estado do Rio de Janeiro foi realizada por Antônio Pedro, no Município de Niterói, em 1923. Desde então, não houve relato de nenhum outro caso durante 63 anos, até a reintrodução do vírus em 1986 2,5,6. Após a mesma, ocorreram cinco epidemias de dengue no estado, nos anos de: 1986/1987, 1990/1991, 1995, 1998 e $2001 / 2002$.

O DEN-1 foi isolado em abril de 1986, durante um surto de doença exantemática no Município de Nova Iguaçu, Rio de Janeiro 7. Além desse surto em Nova Iguaçu, nesse ano foram diagnosticados os primeiros casos no Município do Rio de Janeiro, e houve então o início da disseminação do sorotipo DEN-1 1 . 
A epidemia no Estado do Rio no biênio 1986/1987 superou qualquer outro país americano ${ }^{8}$, e já nesse ano o vetor era encontrado em praticamente todos os estados brasileiros 9 . A grande circulação de pessoas na Região Metropolitana do Rio de Janeiro facilitou a rápida difusão do DEN-1 6, sendo este o responsável pelo biênio epidêmico 1986/1987 10 .

O sorotipo DEN-2 foi introduzido em abril de 1990, durante um período de alta atividade do vírus DEN-1, na cidade de Niterói 11. Na primeira alça do biênio epidêmico 1990/1991, houve circulação concomitante de DEN-1 e DEN-2, com predomínio do DEN-1 6. A partir de agosto de 1990 até 1991, na segunda alça do biênio epidêmico, observou-se já um importante aumento da incidência de dengue pelo sorotipo DEN-2, associado ao surgimento de casos graves como a febre hemorrágica do dengue (FHC) e a síndrome de choque do dengue (SCD) 6,12.

Ocorreram novas epidemias de menor monta nos anos de 1995 e 1998, porém caracterizadas pela rápida difusão do vírus para regiões do estado até então não acometidas, principalmente a Região do Médio Paraíba e a importante área turística das Baixadas Litorâneas 6 .

O DEN-3, sorotipo mais virulento, foi isolado pela primeira vez, no Estado do Rio de Janeiro, no Município de Nova Iguaçu, em dezembro de 2000 13. A partir de março de 2001, ocorreu co-circulação do DEN-3 e do DEN-2, com predomínio deste último (primeira alça do biênio), porém, a partir de dezembro do mesmo ano desencadeou-se explosiva epidemia, na segunda alça do processo. Essa foi a maior epidemia registrada no estado, com 368.460 casos, dos quais 177.919 ocorreram no Município do Rio de Janeiro 13.

As medidas de controle do dengue têm sido baseadas exclusivamente no controle químico do vetor, em detrimento de importantes fatores moduladores da dinâmica de transmissão da doença, especialmente os relacionados a determinantes sociais, como ocupação espacial e condições de vida. Portanto, a cada introdução de um novo sorotipo ocorre nova epidemia. Observa-se um padrão caracterizado por biênios epidêmicos intercalados por períodos de baixa incidência. Essa redução da incidência mais provavelmente se deve ao declínio da população de suscetíveis do que à efetividade das medidas de controle 1 .

Nos biênios epidêmicos que sucederam a introdução dos sorotipos, observa-se uma primeira onda epidêmica, em função da densidade vetorial e da população suscetível, seguida por um período de redução drástica dos casos, coincidente com os meses frios, porém ainda com circulação viral. Com a chegada do verão, ocorre nova onda epidêmica, que assume então caráter explosivo, devido aos altos índices de infestação vetorial e à disseminação do novo sorotipo recém-introduzido ${ }^{14}$.

A manutenção da endemia nos grandes centros urbanos deve-se a uma complexa rede de fatores inerentes às formas de organização da vida nas metrópoles. O saneamento básico, particularmente o abastecimento de água e a coleta de lixo, mostra-se insuficiente ou inadequado nas periferias, gerando um aumento do número de criadouros potenciais do A. aegypti. $\mathrm{O}$ sistema produtivo industrial moderno produz uma grande quantidade de recipientes descartáveis, cujo destino inadequado também contribui consideravelmente para a proliferação do vetor. Outro importante fator é o aumento exorbitante da produção de veículos automotores, e o conseqüente destino inadequado de pneus usados. É importante ainda a disseminação passiva desses transmissores, sob a forma de ovos ou larvas, em recipientes contendo água, como vasos de flores, plantas aquáticas e outros 15.

Os fatores envolvidos no aumento da transmissão de dengue no Brasil são complexos e não totalmente compreendidos. Uma das razões é que o Programa de Controle de Vetores baseouse em métodos verticais, buscando a eliminação do mosquito por meio de inseticidas, o que mostrou pouco impacto na diminuição da proliferação do vetor. A população teve o papel de mera expectadora, mantendo-se na dependência de ações previamente definidas ${ }^{2}$.

A compreensão da dinâmica espacial da doença e suas relações com o contexto social, o qual favorece a permanência do vetor em áreas urbanas e sua capacidade de transmissão, oferece subsídios para a implementação de estratégias mais eficazes de controle do mosquito.

Este estudo buscou analisar a distribuição espacial da epidemia de dengue no Estado do Rio de Janeiro em 2002, e suas relações com as variáveis sócio-demográficas, mediante a utilização de técnicas de análise espacial, como o mapeamento por meio de ferramentas de SIG (Sistema de Informações Geográficas), análise espacial e modelagem estatística. Desse modo, foi realizada uma análise ecológica de dados censitários e epidemiológicos da doença, com o intuito de verificar possíveis associações e melhor compreender o contexto de produção da doença.

\section{Materiais e métodos}

O Estado do Rio de Janeiro, situado na Região Sudeste do Brasil, está dividido em oito regiões político-administrativas e 92 municípios, possui uma população de 15.383.407 habitantes e uma 
área total de $43.696 .054 \mathrm{~km}^{2}$ (Instituto Brasileiro de Geografia e Estatística - IBGE; http://www. ibge.gov.br, acessado em 20/Jun/2006).

O método epidemiológico de análise utilizado foi o desenho ecológico analítico, em que se avaliou a associação entre o nível de exposição médio (variáveis sócio-demográficas) e a incidência do dengue em áreas geográficas delimitadas, como os municípios.

As unidades de análise foram 90 dos $92 \mathrm{mu}$ nicípios do estado, com exceção de Varre-sai e Mesquita. Foi criado um mapa sem esses dois municípios, valendo-se de uma base cartográfica, referente ao ano de 2002 (Departamento de Informática do SUS. Malha municipal digital do Brasil - situação em 2001. http:/ /www.datasus. gov.br, acessado em 20/Jun/2006). O Município de Varre-sai foi excluído da análise por não apresentar nenhum caso notificado na grande epidemia de 2002, provavelmente devido à subnotificação, visto que este mesmo município apresentou incidência de 12,59 casos/100 mil habitantes no início do biênio epidêmico, em 2001 16. O Município de Mesquita foi excluído da análise, pois este sofreu emancipação apenas em 2001, sendo que no estudo foram utilizadas variáveis do Censo Demográfico 2000 (IBGE; http://www. ibge.gov.br), no qual ainda não constam dados sobre Mesquita. Assim, para o ano de 2002, esse município foi agregado ao seu município de origem, Nova Iguaçu.

Foram estudados os casos notificados de dengue no Estado do Rio de Janeiro, no ano de 2002, usando-se o Sistema de Informação de Agravos de Notificação (SINAN) 16. Foi utilizado um banco de dados com informações sociodemográficas, referentes ao Censo Demográfico 2000, obtidas por meio do IBGE (http://www. ibge.gov.br), e informações relacionadas à assistência à saúde, obtidas no DATASUS (Departamento de Informática do SUS. Informações em saúde. http://www.datasus.gov.br, acessado em 20/Jun/2006). As variáveis independentes estudadas foram: expectativa de vida ao nascer; proporção de aumento da expectativa de vida ao nascer (1991-2000); taxa de crescimento populacional; densidade demográfica; proporção de população urbana; percentual de população com água canalizada; percentual de população com esgotamento sanitário; proporção de domicílios com esgotamento sanitário; percentual de população com coleta de lixo; percentual de população sem água canalizada, esgotamento sanitário e coleta de lixo; taxa de analfabetismo; percentual de crianças de 7 a 14 anos fora da escola; PIB per capita; percentual de pobreza; percentual de indigência; Índice de Gini; razão de dependência; índice de envelhecimento; percentual de áreas degradadas; percentual de áreas urbanas; quantidade de crianças entre 10 e 14 anos; percentual de idosos que vivem sós; percentual de cobertura vacinal; percentual de cobertura do Programa Saúde da Família (PSF) e percentual de cobertura do pré-natal.

Foram utilizadas as seguintes técnicas de análise espacial para verificação de associação entre a incidência de dengue e as variáveis explicativas em nível de grupo: mapeamento da incidência de dengue no ano de 2002; análise estatística espacial, para verificação de autocorrelação espacial por meio do Índice Global de Moran; análise exploratória do desfecho e das variáveis sóciodemográficas; modelagem estatística para busca do melhor modelo explicativo para o desfecho; mapeamento das variáveis do modelo final e dos resíduos do modelo.

Para a modelagem foi utilizado o modelo de regressão linear múltipla. Os programas utilizados foram: Epi Info 2000 (Centers for Disease Control and Prevention, Atlanta, Estados Unidos), ArcView 3.3 (Environmental Systems Research Institute Inc., http://www.esri.com/software/ arcview/) e S-Plus 2000 (Mathsoft Inc., Seattle, Estados Unidos).

Calculou-se a taxa de incidência de dengue por municípios do estado, em 2002, e então foi realizado o mapeamento usando-se o ArcView 3.3. Realizou-se a análise estatística dos dados espaciais, por meio da construção de uma matriz de vizinhança com o critério de vizinhos de primeira ordem para os municípios, e aplicação do Índice Global de Moran, que verifica se as áreas geograficamente vizinhas apresentam maior semelhança quanto às variáveis estudadas do que o esperado ao acaso, sendo o índice positivo para correlação direta e negativo para correlação inversa.

Realizou-se análise exploratória das variáveis independentes, por meio da matriz de correlação (coeficiente de Spearman), para avaliação da direção e magnitude das correlações entre desfecho e variáveis. Das 25 variáveis existentes no banco de dados, 13 apresentaram correlação significativa com o desfecho em nível de 5\%, tendo sido então selecionadas para a modelagem.

Foi empregada uma transformação do tipo logaritmo neperiano para a variável dependente, com o objetivo de melhor aproximar a distribuição da incidência a uma distribuição normal, visando a realização da regressão linear.

Após a modelagem, realizada utilizando-se o modelo de regressão linear multivariada, foi realizado o mapeamento dos resíduos do modelo final, para verificação do pressuposto de independência espacial dos resíduos. Foi realizada também aplicação do Índice Global de Moran para quantificação da autocorrelação existente 
nos resíduos de cada modelo. A qualidade do ajuste do modelo é verificada por meio da análise de resíduos com base no Índice Global de Moran 17.

Quando a autocorrelação espacial das variáveis está presente, as estimativas do modelo de regressão devem incorporar esta estrutura espacial, pois a dependência entre as observações altera o poder explicativo do modelo. Caso os resíduos do modelo apresentem manutenção da estrutura de dependência espacial, o uso de um modelo de regressão espacial torna-se adequado, pois capta esta dependência das variáveis 18 .

Porém, no presente estudo, apesar de ter sido encontrada autocorrelação espacial significativa tanto para a variável desfecho quanto para as variáveis do modelo final da regressão linear, não se tornou necessária a inclusão de um condicional espacial na modelagem. Isso se justifica porque quando os resíduos do modelo final não apresentam manutenção do padrão de dependência espacial, a inclusão de um condicional espacial introduziria graus adicionais de liberdade que nada acrescentariam aos resultados, além de prejudicar o princípio de parcimônia da modelagem.

\section{Resultados}

A análise exploratória das correlações entre as variáveis, realizada por meio da matriz de correlação, mostrou que 13 variáveis apresentaram correlação estatisticamente significativa, em nível de $5 \%$, com a incidência de dengue em 2002: taxa de crescimento populacional $(r=0,255)$; densidade demográfica $(r=0,482)$; proporção de população urbana $(r=0,452)$; percentual de população com água canalizada $(\mathrm{r}=-0,269)$; percentual de população com esgotamento sanitário $(\mathrm{r}=0,348)$; percentual de população com coleta de lixo (r
$=0,241)$; taxa de analfabetismo $(r=-0,383)$; percentual de pobreza (renda inferior a $\mathrm{R} \$ 151,00)(\mathrm{r}$ $=-0,271$ ); percentual de indigência (renda inferior a $\mathrm{R} \$ 80,00)(\mathrm{r}=-0,262)$; razão de dependência $(\mathrm{r}=-0,305)$; percentual de áreas urbanas $(\mathrm{r}=$ $0,471)$; percentual de cobertura vacinal $(r=0,193)$ e percentual de cobertura do PSF $(r=-0,406)$.

$\mathrm{Na}$ análise de regressão linear multivariada realizada com as 13 variáveis selecionadas, o modelo final obtido continha três variáveis explicativas para a variabilidade da incidência de dengue na epidemia de 2002 no Estado do Rio de Janeiro: proporção de população urbana, percentual de população com água canalizada e percentual de cobertura do PSF. O coeficiente de determinação R2 encontrado no modelo indica que $30,2 \%$ da variabilidade total das taxas de incidência de dengue na epidemia de 2002 podem ser explicadas por essas variáveis (Tabela 1).

O mapeamento da incidência de dengue em 2002 revelou aglomerados de incidência nas Regiões Metropolitana e Médio Paraíba (Figura 1). A Região Metropolitana está situada no sudeste, enquanto a Região do Médio Paraíba localiza-se no sudoeste do Estado do Rio de Janeiro.

A Figura 2 apresenta o mapa de resíduos do modelo final. A concentração de resíduos positivos ou negativos em áreas do mapa representa a permanência da autocorrelação espacial 17. Nos resíduos do modelo foi encontrado Índice Global de Moran muito baixo $(-0,001)$ e não significativo $(p=0,886)$, o que representa a inexistência de autocorrelação espacial (Tabela 2). Isso significa que, apesar de ter sido observado padrão de dependência espacial para a variável desfecho, a estrutura espacial existente nos dados foi explicada satisfatoriamente pelas variáveis do modelo final, não sendo, portanto, necessário incluir um condicional espacial para melhoria da qualidade de ajuste do modelo. Mantêm-se, portanto, os resultados do modelo

Modelo de regressão linear multivariada da incidência de dengue e das variáveis proporção de população urbana, percentual de população com água canalizada e percentual de cobertura do Programa Saúde da Família (PSF). Estado do Rio de Janeiro, Brasil, 2002.

\begin{tabular}{lcccc}
\hline Variáveis & Coeficiente & Erro-padrão & Teste t & Valor de p \\
\hline Intercepto & 8,4340 & 1,5320 & 5,5050 & 0,0000 \\
População urbana & 0,0244 & 0,0060 & 3,9710 & 0,0000 \\
População com água canalizada & $-0,0381$ & 0,0160 & $-2,3770$ & 0,0200 \\
Cobertura do PSF & $-0,0072$ & 0,0040 & $-2,0170$ & 0,0470 \\
\hline
\end{tabular}

$\mathrm{R} 2=0,302$. 
Figura 1

Mapas: a) incidência de dengue no Estado do Rio de Janeiro, Brasil, em 2002; b) proporção de população urbana; c) percentual de população com água canalizada; d) percentual de cobertura do Programa Saúde da Família (PSF).

a)

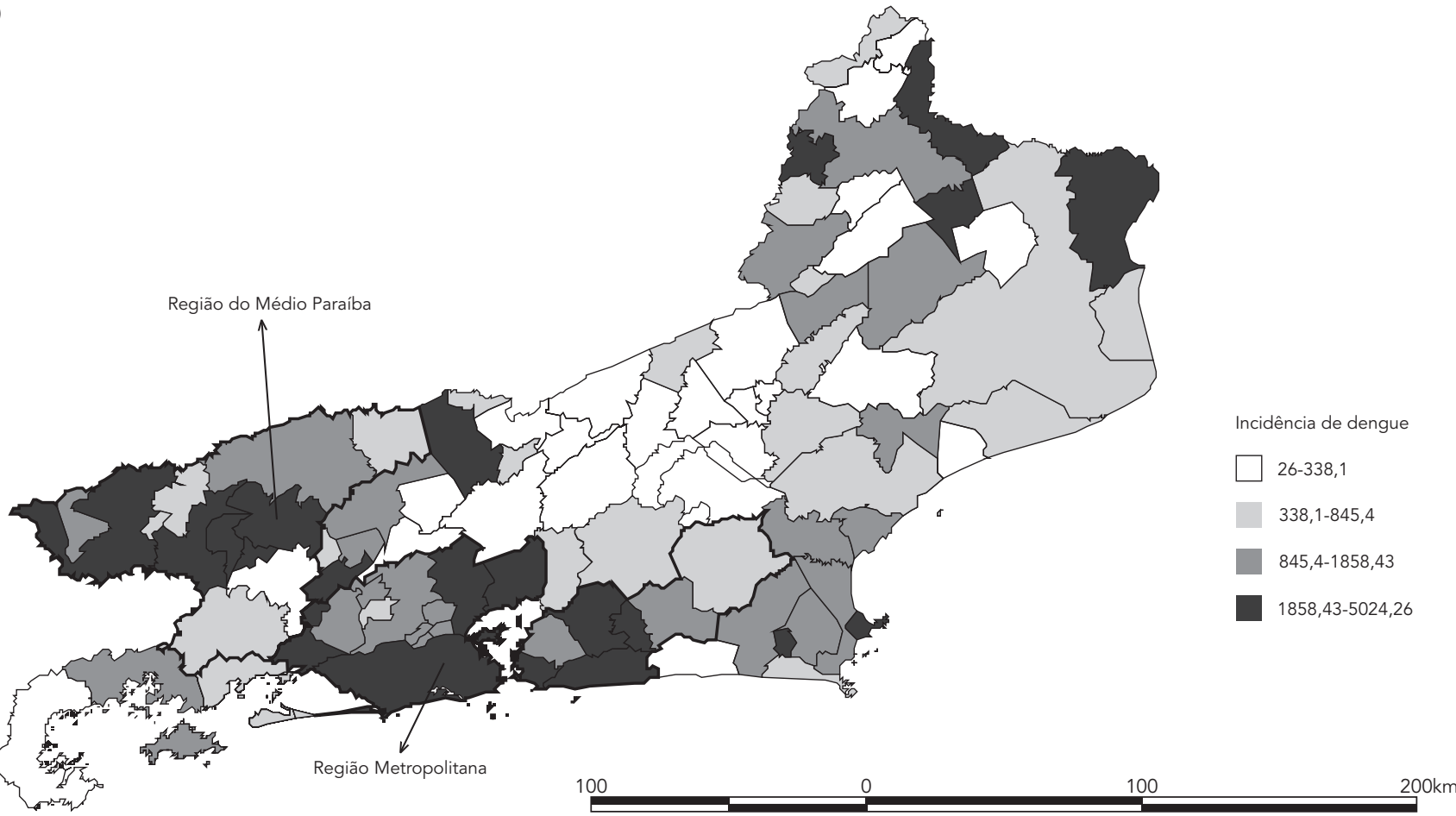

b)

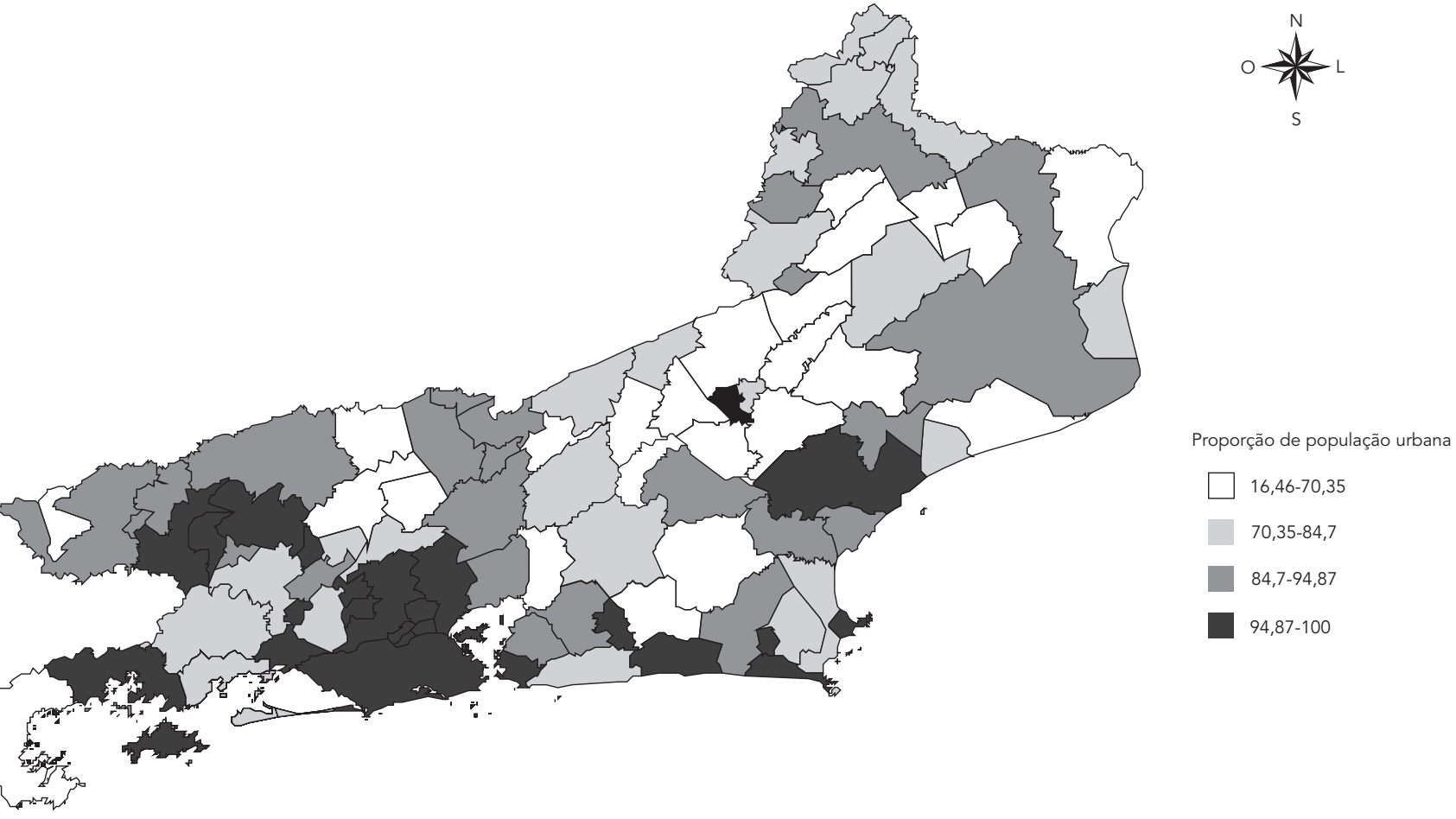

(continua) 
c)

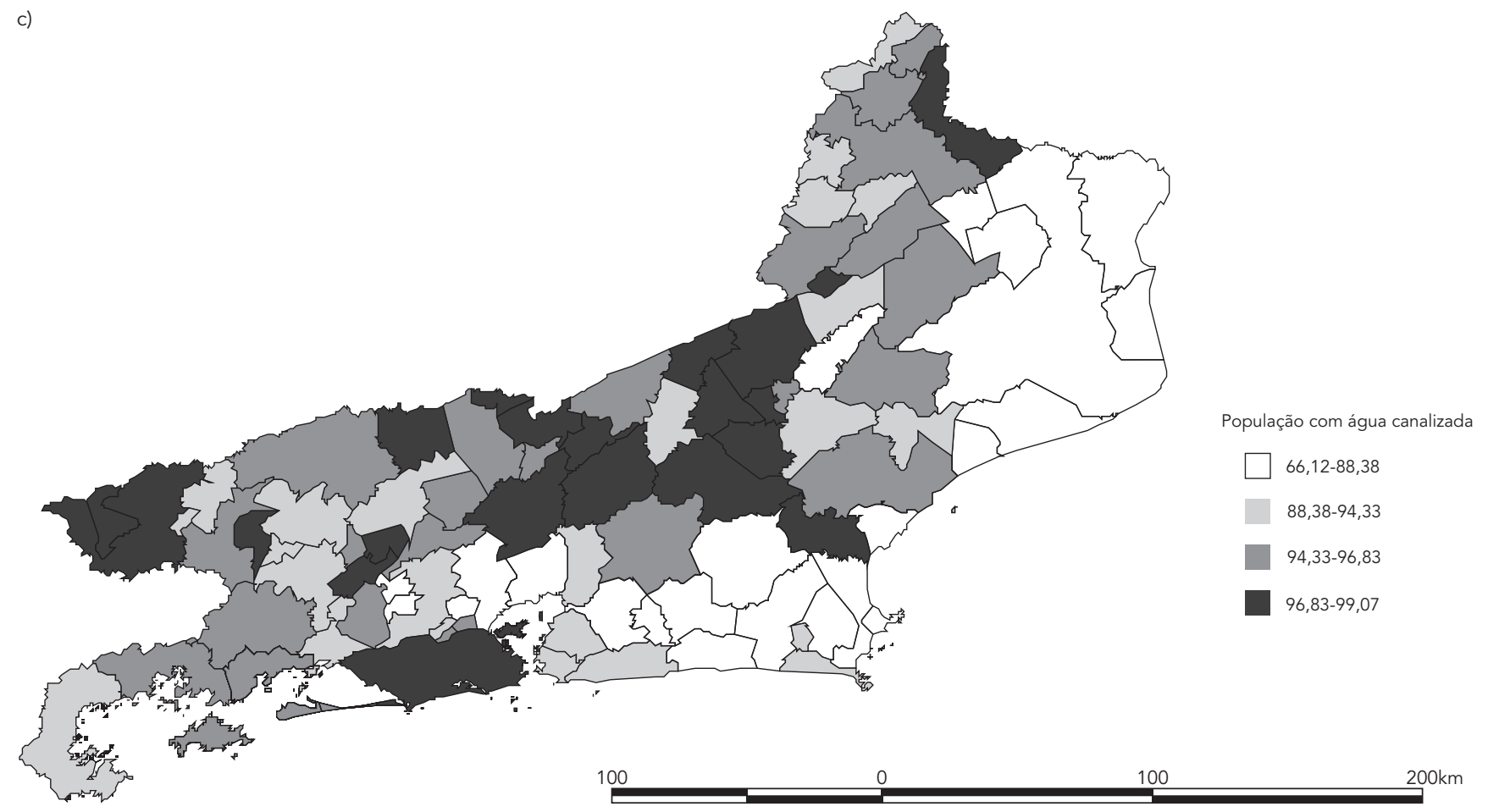

d)

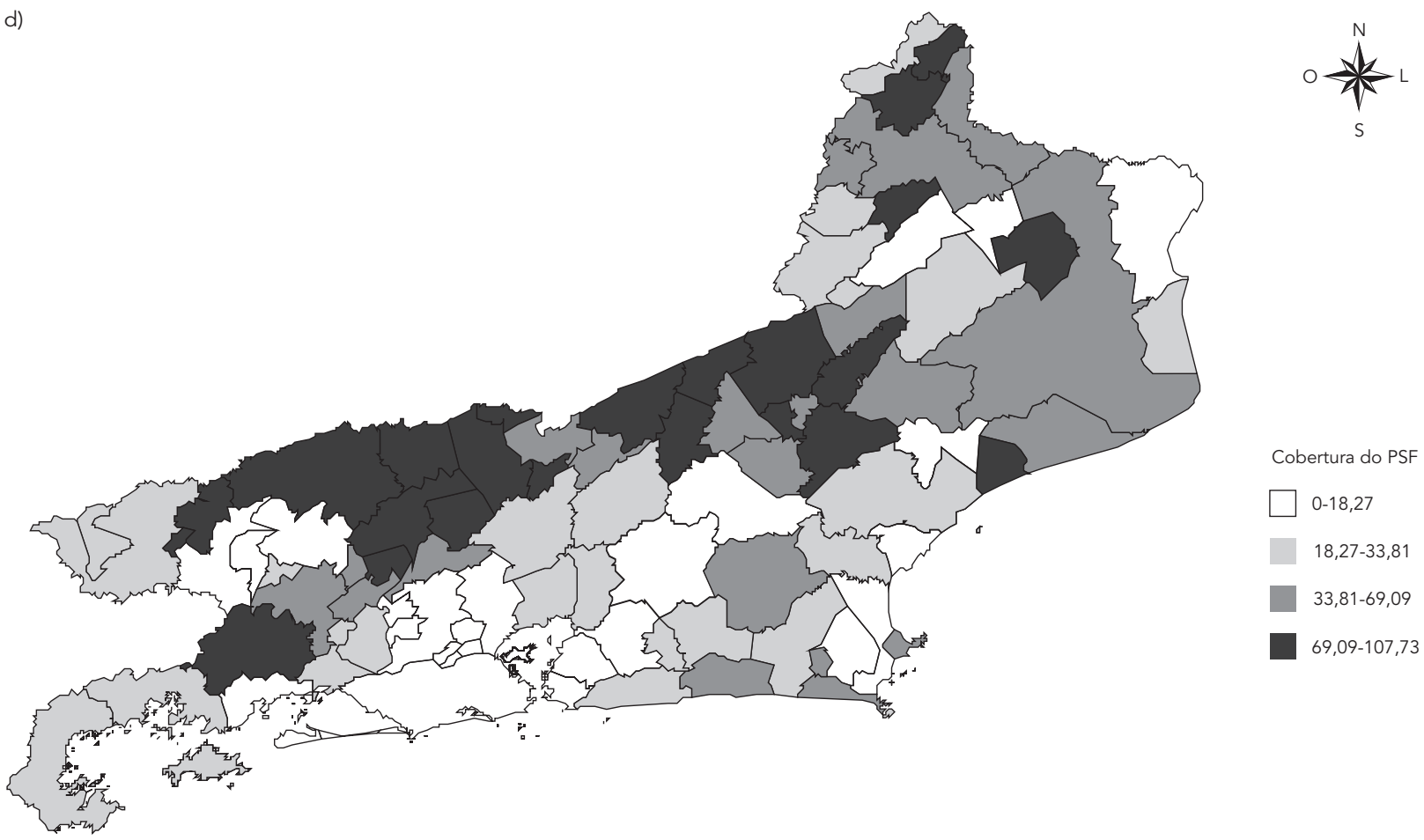




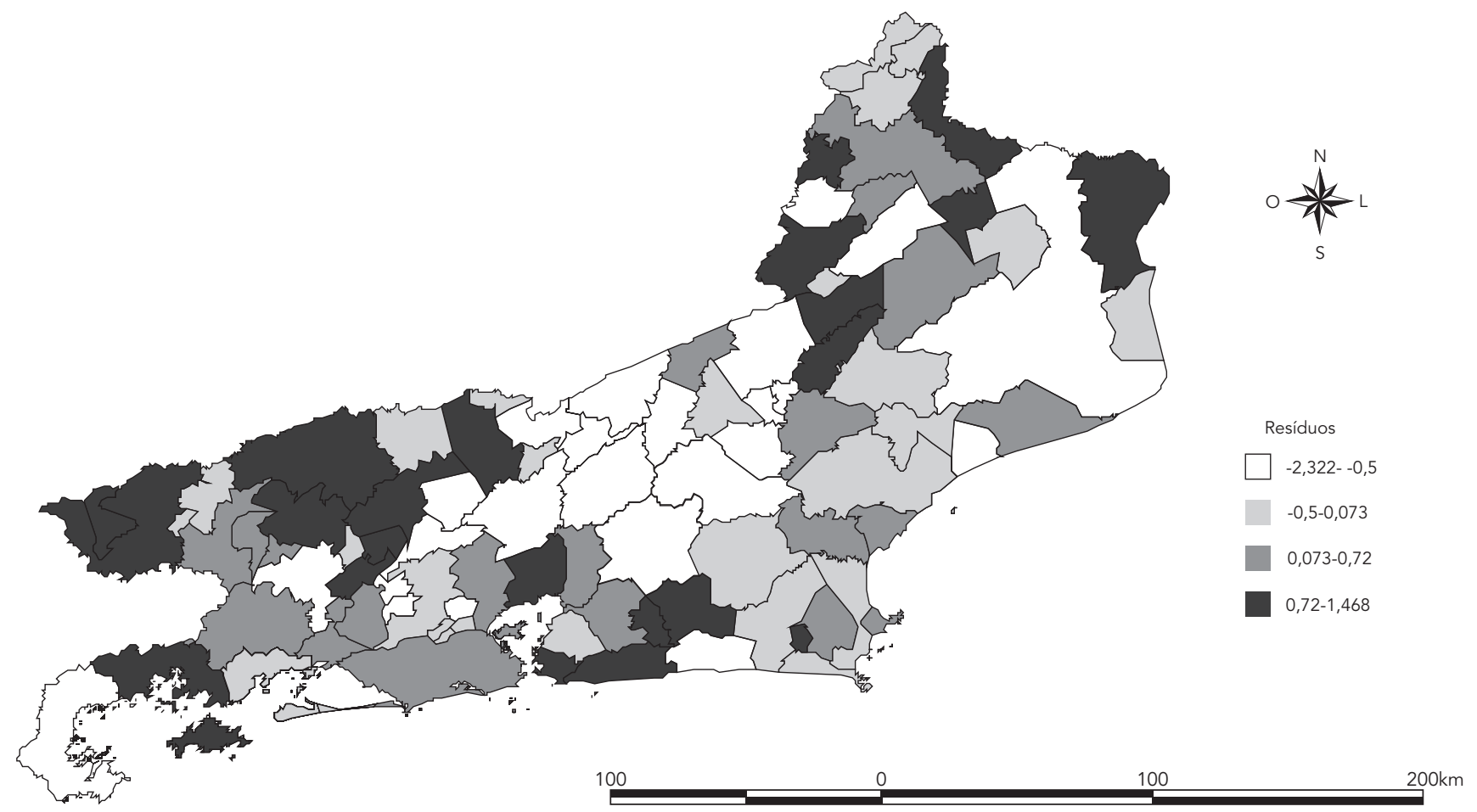

Tabela 2

Índice Global de Moran e níveis de significância.

\begin{tabular}{lcc}
\hline Variáveis & Índice Global de Moran & Valor de p \\
\hline Incidência de dengue em 2002 & 0,1726 & 0,00480 \\
Proporção de população urbana & 0,2444 & 0,00008 \\
Percentual de população com água canalizada & 0,2504 & 0,00005 \\
Percentual de cobertura do Programa Saúde da Família & 0,3160 & $<0,00000$ \\
\hline
\end{tabular}

de regressão linear, pois se não há manutenção da estrutura espacial nos resíduos, a utilização de um condicional espacial geraria a inclusão de graus adicionais de liberdade, e com isto um modelo final menos parcimonioso.

\section{Discussão}

Constatou-se que, das 25 variáveis estudadas, três podem explicar $30,2 \%$ da variabilidade das taxas de incidência de dengue na epidemia de 2002 no Estado do Rio de Janeiro: proporção de população urbana (associação direta), percentual de população com água canalizada e percentual de cobertura do PSF (ambas com associação inversa).

O crescimento acelerado da população urbana propicia grandes fontes de indivíduos suscetíveis e infectados, concentrados em áreas restritas. Esse fato, associado às condições precárias de saneamento, moradia e fatores 
culturais próprios dos grandes centros urbanos, proporcionam condições ecológicas favoráveis à transmissão do vírus do dengue ${ }^{19}$. Portanto, é compreensível que localidades com maior proporção de população urbana possam apresentar incidências elevadas de dengue.

As mudanças demográficas ocorridas nos países subdesenvolvidos a partir da década de 60 resultaram no crescimento desordenado das cidades. O saneamento básico, especialmente o abastecimento de água e a coleta de lixo, mostra-se insuficiente ou inadequado. Portanto, as ações de prevenção demandam envolvimento de diversos setores da sociedade, no que tange à questão da melhoria das condições de urbanização, habitação, saneamento e educação ${ }^{2}$. Além disso, a irregularidade no fornecimento de água canalizada pode implicar situações favoráveis à procriação do vetor ${ }^{20}$. Com a falta de abastecimento de água, torna-se necessário armazenála precariamente em depósitos improvisados para água potável. Isso provoca a proliferação de criadouros potenciais do A. aegypti ${ }^{2}$.

Em estudo anterior, realizado numa localidade urbana do Sudeste do Brasil em 1998 19, foi encontrada maior incidência de dengue em áreas onde os serviços de saneamento básico eram mais precários. No entanto, não foi encontrada correlação entre a incidência de dengue e o percentual de domicílios com abastecimento de água canalizada. Encontrou-se correlação apenas com a coleta de lixo e a rede de coleta de esgotos. Em estudo anterior, de 1996, também na Região Sudeste, foi encontrada, em localidades com altos índices de incidência da doença, participação relativamente grande de recipientes para armazenamento de água na composição do índice larvário de A. aegypti 21.

O A. aegypti tem uma acentuada preferência por recipientes contendo água relativamente limpa. Dentre os recipientes de caráter permanente, destacam-se os reservatórios domiciliares de água para consumo humano. Esses propiciam excelentes condições para a criação do vetor, contribuindo para a manutenção de populações do culicídeo, mesmo em períodos não favoráveis, como os de baixas precipitações 22 .

Os achados do presente estudo revelaram correlação inversa entre o percentual de população com água canalizada e a incidência de dengue em 2002, ou seja, que o fato de ter água canalizada poderia ser um fator protetor para dengue, pois desta forma não se torna necessário estocar água para consumo em reservatórios.

Os aglomerados urbanos modernos apresentam, nos países pobres, deficiências de saneamento básico, habitação, educação e segurança pública. Estudos anteriores, realizados em diversas capitais brasileiras, também evidenciaram associações entre risco de ocorrência de dengue e níveis sócio-econômicos. Caiaffa et al. ${ }^{23}$, para a cidade de Belo Horizonte, Minas Gerais; Siqueira et al. ${ }^{24}$, para a cidade de Goiânia, Goiás; Medronho 25, para a cidade do Rio de Janeiro; e Costa \& Natal 19, para a cidade de São José do Rio Preto, São Paulo mostraram haver associação positiva entre maiores riscos de transmissão de dengue e piores níveis socioeconômicos da população. Em contrapartida, Mondini \& Chiaravalloti Neto 26, em estudo realizado também na cidade de São José do Rio Preto, no período de setembro de 1994 a agosto de 2002, encontraram resultados concordantes com os de Costa \& Natal 19 apenas para um pequeno período, de setembro de 1994 a agosto de 1995. Nesse último caso, a ausência de associação entre risco de ocorrência de dengue e níveis sócio-econômicos na maioria dos anos estudados, mostra que esta é uma questão bastante controversa, e que precisa ser mais bem explorada. Inclusive, é provável que dependa da realidade de cada município. É importante que sejam verificadas a relação espacial entre a transmissão de dengue e outras variáveis, como o grau de imunidade da população; a efetividade das medidas de controle; o grau de infestação pelo vetor; os hábitos e atitudes da população, entre outros 26.

O PSF, criado com o propósito de reorganizar os serviços de saúde e melhorar a qualidade de vida da população, prioriza as ações de prevenção de doenças, promoção e recuperação da saúde, de forma integral e contínua 27. Em 3 de janeiro de 2002, o Ministério da Saúde publicou a Portaria $n^{\circ} .44 / G M$, que definiu e estabeleceu as atribuições dos agentes comunitários de saúde em relação à prevenção e controle do dengue, conjugando as ações do PSF e do Programa de Controle do Vetor (PCV) e integrando os dois programas 28.

Num estudo comparativo entre os resultados das ações de controle do PSF e do PCV 27, observou-se que as ações de controle do dengue desenvolvidas no âmbito do PSF e do PCV tiveram efetividade semelhante, permitindo a conclusão de que a integração dos dois programas não só é completamente viável como também permite a otimização de recursos ao evitar duplicidade de visitas, além de possibilitar maior envolvimento da comunidade no controle do dengue. Os agentes comunitários de saúde tratam de problemas de saúde de forma ampla e atuam como facilitadores na relação entre os serviços de saúde e a população, o que resulta numa maior adesão desta às atividades de prevenção desenvolvidas por estes agentes. Foram observadas mudanças 
significativas em termos de ganho de conhecimento pela população em relação às medidas de controle do dengue, além de considerável diminuição dos recipientes de acúmulo de água na comunidade.

Encontrou-se, no presente estudo, correlação inversa entre o percentual de cobertura pelo PSF e a incidência de dengue, ou seja, que uma população assistida pelo programa possivelmente estaria menos vulnerável ao dengue.

É possível concluir que uma atuação integrada dos agentes de controle de vetores com as unidades básicas de saúde, e uma articulação com o PSF, poderiam trazer inúmeros benefícios, como o aumento da adesão da população às práticas preventivas, melhoria do vínculo do serviço com a comunidade e promoção da integração do controle do dengue com as demais atividades do SUS 27 .

Dentro dessa perspectiva, este estudo reforça a idéia de que outras formas de atuação, como o trabalho desenvolvido pelos agentes comunitários de saúde, podem dar bons resultados e apresentarem-se como alternativas viáveis. Porém, é importante destacar as limitações da atuação do serviço de saúde no controle do dengue, pois a resolução deste problema deve ser pensada a partir da integração com áreas como saneamento, habitação e educação, além da necessária melhoria das condições de vida da população 29 .

O presente estudo, por tratar-se de uma abordagem ecológica, apresenta limitações inerentes à sua metodologia, com relação à inferência e ge- neralização dos achados. Por esse motivo, não se pode concluir, apenas com base nesses achados, que o PSF deva ser implantado em todas as áreas, visando a um controle efetivo do dengue, nem se pode afirmar que a adequação e canalização da rede de abastecimento de água tenham importância maior para o controle do dengue do que outros fatores relacionados ao saneamento básico, como a coleta de lixo e o esgotamento sanitário. Além disso, não necessariamente todas as áreas de elevada urbanização estariam associadas a maiores incidências de dengue.

Apesar dessa limitação, os achados deste estudo são úteis no levantamento de possíveis fatores de risco e proteção para a difusão do dengue no espaço urbano. É possível, por meio desses achados, indicar que a urbanização e a falta de acesso à água canalizada seriam fatores que possibilitariam um incremento de risco para a ocorrência de casos de dengue, e ainda considerar que o PSF pode ser um importante facilitador das estratégias de controle do vetor. No entanto, torna-se necessária uma investigação mais individualizada de cada um desses fatores, além de outros relacionados aos aspectos sócio-demográficos e ambientais, para que se possa alcançar uma melhor capacidade explicativa e uma melhor compreensão da dinâmica espacial do agravo. Estes são subsídios essenciais para permitir o levantamento de prioridades e das localidades que inspiram maior atenção, visando a um maior impacto das medidas de controle do vetor sobre a saúde da população. 


\section{Resumo}

Este estudo analisou a epidemia de dengue em $2002 e$ o contexto sócio-demográfico do Estado do Rio de Janeiro, Brasil, por meio de técnicas de análise espacial e de modelagem estatística. Calculou-se a taxa de incidência, segundo casos de dengue notificados e residentes no estado em 2002. Foram avaliadas associações entre tal incidência e variáveis sócio-demográficas, $e$ a autocorrelação espacial usando-se o Índice Global de Moran, o qual revelou dependência espacial tanto para a variável desfecho quanto para as independentes. Foi utilizado o modelo de regressão linear multivariada. As variáveis: proporção de população urbana, percentual de população com água canalizada e percentual de cobertura de Programa Saúde da Família (PSF), explicaram $30,2 \%$ da variabilidade total das taxas de incidência para essa epidemia. Os resíduos não revelaram autocorrelação espacial. As associações encontradas se deram na direção esperada e tais achados são corroborados por outros estudos, que encontraram maiores incidências de dengue em localidades caracterizadas pela crescente urbanização e por déficit na rede de canalização e abastecimento de água, e que também evidenciaram o PSF como importante facilitador das estratégias de controle do vetor.

Dengue; Surtos de Doenças; Análise Espacial

\section{Colaboradores}

T. R. A. Teixeira e R. A. Medronho contribuíram em todo o processo de elaboração do artigo.

\section{Agradecimentos}

Os autores agradecem à Prof. Rejane Sobrino Pinheiro e ao João Faria de Figueiredo, do Instituto de Estudos em Saúde Coletiva, da Universidade Federal do Rio de Janeiro e ainda ao Sr. Gualberto Teixeira Filho, da Secretaria de Estado de Saúde do Rio de Janeiro, pela cessão de dados e colaboração com o estudo.

\section{Referências}

1. Teixeira MG, Costa MCN, Barreto ML, Mota E. Dengue and dengue hemorrhagic fever epidemics in Brazil: what research is needed based on trends, surveillance, and control experiences? Cad Saúde Pública 2005; 21:1307-15.

2. Tauil PL. Urbanização e ecologia do dengue. Cad Saúde Pública 2001; 17 Suppl:S99-102.

3. Penna MLF. Um desafio para a saúde pública brasileira: o controle do dengue. Cad Saúde Pública 2003; 19:305-9.

4. Marques AC. Sobre a viabilidade atual de erradicação do Aedes aegypti no controle da Febre Amarela no Brasil. Rev Bras Malariol Doenças Trop 1985; 37:37-46.

5. Pedro A. Dengue em Nichteroy. Brasil Med 1923; 1:173-7.

6. Nogueira RMR, Miagostovich MP, Schatzmayr HG, Santos DFB, Araújo ESM, Filippis AMB, et al. Dengue in the State of Rio de Janeiro, Brazil, 1986-1998. Mem Inst Oswaldo Cruz 1999; 94:297-304.
7. Schatzmayr HG, Nogueira RMR, Travassos-da-Rosa APA. An outbreak of dengue virus at Rio de Janeiro - 1986. Mem Inst Oswaldo Cruz 1986; 81:245-6.

8. Donalisio MR. O dengue no espaço habitado. São Paulo: Editora Hucitec; 1999.

9. Neves DP, Melo AL, Genaro O, Linardi PM. Culicídeos. In: Neves DP, Melo AL, Genaro O, Linardi PM, organizadores. Parasitologia humana. 9a Ed. São Paulo: Editora Atheneu; 1995. p. 383-401.

10. Marzochi KBF. Dengue: a mais nova endemia de estimação. Cad Saúde Pública 1987; 32:137-41.

11. Nogueira RMR, Miagostovich MP, Lampe E, Schatzmayr HG. Isolation of dengue vírus type 2 in Rio de Janeiro. Mem Inst Oswaldo Cruz 1990; 85:253.

12. Fundação Nacional de Saúde, Ministério da Saúde. Programa nacional de controle da dengue. Brasília: Ministério da Saúde; 2002. 
13. Nogueira RMR, Miagostovich MP, Filippis AMB, Schatzmayr HG, Pereira MAS. Dengue virus type 3 in Rio de Janeiro, Brazil. Mem Inst Oswaldo Cruz 2001; 96:925-6.

14. Casali CG, Pereira MRR, Santos LMJG, Passos MNPP, Fortes BPMD, Valencia LIO, et al. A epidemia de dengue/dengue hemorrágico no município do Rio de Janeiro, 2001/2002. Rev Soc Bras Med Trop 2004; 37:296-9.

15. Gubler DJ. Dengue and dengue hemorrhagic fever: its history and resurgence as a global health problem. In: Gubler DJ, Kuno G, editors. Dengue and dengue and hemorragic fever. New York: $\mathrm{CAB}$ International; 1997. p. 1-22.

16. Secretaria de Estado de Saúde do Rio de Janeiro. Quadro demonstrativo de casos notificados de dengue no Estado do Rio de Janeiro. Rio de Janeiro: Secretaria de Estado de Saúde do Rio de Janeiro; 2006.

17. Lima MLC, Ximenes RAA, Souza ER, Luna CF, Albuquerque MFPM. Análise espacial dos determinantes socioeconômicos dos homicídios no Estado de Pernambuco. Rev Saúde Pública 2005; 39:176-82.

18. Druck S, Carvalho MS, Câmara G, Monteiro AMV. Análise espacial de dados geográficos. Planaltina: Empresa Brasileira de Pesquisa Agropecuária; 2004.

19. Costa AIP, Natal D. Distribuição espacial da dengue e determinantes socioeconômicos em localidade urbana no Sudeste do Brasil. Rev Saúde Pública 1998; 32:232-6.

20. Oliveira RM, Valla VV. Living conditions and life experiences of working-class groups in Rio de Janeiro: rethinking dengue control and popular mobilization. Cad Saúde Pública 2001; 17 Suppl:S77-88.

21. Costa AIP. Identificação de unidades ambientais urbanas como condicionantes da ocorrência de Aedes aegypti (Díptera: Culicidae) e de dengue na cidade de São José do Rio Preto, SP, em 1995 [Dissertação de Mestrado]. São Paulo: Faculdade de Saúde Pública, Universidade de São Paulo; 1996.
22. Forattini OP, Brito M. Reservatórios domiciliares de água e controle do Aedes aegypti. Rev Saúde Pública 2003; 37:676-7.

23. Caiaffa WT, Almeida MC, Oliveira CD, Friche AA, Matos SG, Dias MA, et al. The urban environment from the health perspective: the case of Belo Horizonte, Minas Gerais, Brazil. Cad Saúde Pública 2005; 21:958-67.

24. Siqueira JB, Martelle CM, Maciel IJ, Oliveira RM, Ribeiro MG, Amorim FP, et al. Household survey of dengue infection in Central Brazil: spatial point pattern analysis and risk factors assessment. Am J Trop Med Hyg 2004; 71:646-51.

25. Medronho RA. Geoprocessamento e saúde: uma nova abordagem do espaço no processo saúde doença. Rio de Janeiro: Fundação Oswaldo Cruz; 1995.

26. Mondini A, Chiaravalloti Neto F. Variáveis socioeconômicas e a transmissão de dengue. Rev Saúde Pública 2007; 41:923-30.

27. Chiaravalloti Neto F, Barbosa AAC, Cesarino MB, Favaro EA, Mondini A, Ferraz A, et al. Controle da dengue em uma área urbana do Brasil: avaliação do impacto do Programa Saúde da Família com relação ao programa tradicional de controle. Cad Saúde Pública 2006; 22:987-97.

28. Ministério da Saúde. Portaria no ${ }^{\circ}$ 44/GM. http:// d tr2001.saude.gov.br/bvs/popup/leg/ portarias_psf/portaria_n44_2002.pdf (acessado em 20/Jan/2007).

29. Silva Júnior JB, Siqueira Junior JB, Coelho GE, Vilarinhos PTR, Pimenta-Júnior FG. Dengue in Brazil: current situation and prevention and control activities. Epidemiol Bull 2002; 23:3-6.

Recebido em 21/Jun/2007

Versão final reapresentada em 10/Jan/2008

Aprovado em 15/Jan/2008 